\title{
Markers of acute kidney injury in children undergoing hematopoietic stem cell transplantation
}

\author{
Monika Augustynowicz ${ }^{1, A-D}$, Agnieszka Bargenda-Lange ${ }^{1, B-D}$, \\ Krzysztof Kałwak ${ }^{2, A, E, F}$, Danuta Zwolińska ${ }^{1, E, F}$, Kinga Musiał ${ }^{1, A-F}$ \\ ${ }^{1}$ Department of Pediatric Nephrology, Wroclaw Medical University, Poland \\ ${ }^{2}$ Department of Bone Marrow Transplantation, Oncology and Pediatric Hematology, Wroclaw Medical University, Poland \\ A - research concept and design; B - collection and/or assembly of data; C - data analysis and interpretation; \\ $D$ - writing the article; $E$ - critical revision of the article; $F$ - final approval of the article
}

Address for correspondence

Kinga Musiał

E-mail: kinga_musial@hotmail.com

Funding sources

None declared

Conflict of interest

None declared

Received on 0ctober 29, 2018

Reviewed on November 25, 2018

Accepted on December 13, 2018

Published online on February 11, 2019

Cite as

Augustynowicz M, Bargenda-Lange A, Kałwak K, Zwolińska D, Musiał K. Markers of acute kidney injury in children undergoing hematopoietic stem cell transplantation. Adv Clin Exp Med. 2019;28(8):1111-1118. doi:10.17219/acem/101573

DOI

10.17219/acem/101573

Copyright

Copyright by Author(s)

This is an article distributed under the terms of the

Creative Commons Attribution Non-Commercial License

(http://creativecommons.org/licenses/by-nc-nd/4.0/)

\begin{abstract}
Acute kidney injury (AKI), one of the major complications in children undergoing hematopoietic stem cell transplantation (HSCT), is an independent predictor of the patient's survival and a prognostic factor of progression to chronic kidney disease (CKD). Despite the multifaceted role of AKL, its early diagnosis in the course of HSCT remains a challenge. These difficulties may result from the inefficiency of traditional methods used to assess kidney function, like serum creatinine or estimated glomerular filtration rate. Moreover, the list of potential AKI markers tested in HSCT conditions is limited and does not involve indexes evaluated in the pediatric population. This review summarizes current knowledge on the pathophysiology of AKI developing in the course of HSCT; presents well-known markers of AKI that are potentially applicable in children who have undergone HSCT; discusses the role of new markers in diagnosing AKI and predicting the renal outcome in children undergoing $\mathrm{HSCT}$; and analyzes the prospects for the use of new tools for assessing kidney injury in everyday clinical practice.
\end{abstract}

Key words: chronic kidney disease, nephrotoxicity, acute tubular damage, renal outcome, hematopoietic stem cell transplantation 
Hematopoietic stem cell transplantation (HSCT) is an area of medicine under constant development. In recent years, the number of allogeneic bone marrow transplantations has increased significantly. In addition to hemato-oncological diseases, the indications for HSCT include immunologic, metabolic and genetically determined conditions.

\section{Current methods of assessing kidney function following hematopoietic stem cell transplantation}

As HSCT has become more and more frequently the therapy of choice in children, the number of complications observed has increased. Among them, acute kidney injury (AKI) has become one of the major challenges, as it is an independent predictive factor for patient survival and progression to chronic kidney disease (CKD) ${ }^{1,2}$

Although there are many reviews on HSCT, few of them concern the pediatric population, ${ }^{3-5}$ and none of them concentrate on the nephrological aspects of HSCT.

Moreover, the commonly used classifications of AKI comprise various criteria established by the Risk, Injury, Failure, Loss and End-stage (RIFLE) renal failure classification system, the Acute Kidney Injury Network (AKIN) and the Kidney Disease: Improving Global Outcomes (KDIGO) organization. ${ }^{6-8}$ However, only pRIFLE criteria apply specifically to children (Table 1 ).

Due to the diversity of these classifications, world statistics on the incidence of AKI in children are very inconsistent. As shown in a study by Sutherland et al., the incidence of AKI in a single group of hospitalized children varied significantly depending on the criteria used (51.1\%, $37.1 \%$ and $40.3 \%$ according to pRIFLE, AKIN and KDIGO, respectively). ${ }^{9}$ The AKIN and KDIGO classifications rely on changes in serum creatinine concentration, while pRIFLE criteria are additionally based on decreases in the estimated glomerular filtration rate (eGFR) and urine output (Table 1). Moreover, the fact that the pRIFLE criteria take into account the time frame and diversification of the outcome (reversible injury vs progression to CKD) make them the most useful for children.

It is worth noting that in cases of kidney function deterioration, increases in serum creatinine concentration are significantly delayed. Furthermore, many factors, such as nutrition, hydration, gender, age, and muscle mass, influence the serum concentration of creatinine. Hydration status after HSCT varies depending on the time elapsed from the procedure. During the first few days after HSCT, the treatment protocol assumes a high fluid intake $\left(3 \mathrm{~L} / \mathrm{m}^{2}\right.$ of body surface), resulting in transient hyperfiltration. Moreover, the method of eGFR calculation raises certain doubts. The most commonly applied Schwartz formula overestimates eGFR value, especially when it is higher than $75 \mathrm{~mL} / \mathrm{min} / 1.73 \mathrm{~m}^{2}$. However, attempts to introduce other methods have so far been unsatisfactory. ${ }^{10-12}$ As a consequence, evaluation based on serum creatinine concentration remains the most frequent tool to assess kidney function in children undergoing HSCT, despite its many limitations in terms of sensitivity and specificity for AKI. However, recent reports show preliminary attempts to widen the spectrum of AKI indices by testing markers of inflammation or damage specific to selected compounds of the renal parenchyma.

The purpose of this review was to briefly present current knowledge about the pathophysiology of AKI in the course

Table 1. Comparison of various criteria defining acute kidney injury

\begin{tabular}{|c|c|c|}
\hline Pediatric (p)RIFLE & eGFR & Urine output \\
\hline Risk (Stage 1) & decrease $<25 \%$ & $<0.5 \mathrm{~mL} / \mathrm{kg} / \mathrm{h}$ for $8 \mathrm{~h}$ \\
\hline Injury (Stage 2) & decrease $<50 \%$ & $<0.5 \mathrm{~mL} / \mathrm{kg} / \mathrm{h}$ for $16 \mathrm{~h}$ \\
\hline Failure (Stage 3) & $\begin{array}{c}\text { decrease }<75 \% \\
\text { or }<35 \mathrm{~mL} / \mathrm{min} / 1.73 \mathrm{~m}^{2}\end{array}$ & $\begin{array}{c}<0.3 \mathrm{~mL} / \mathrm{kg} / \mathrm{h} \text { for } 24 \mathrm{~h} \\
\text { or anuria for } 12 \mathrm{~h}\end{array}$ \\
\hline Loss & failure $>4$ weeks & \\
\hline End-stage kidney disease & persistent failure $>3$ months & \\
\hline AKIN criteria & serum creatinine & \\
\hline Stage 1 & $\begin{array}{c}\text { increase } \geq 50 \% \text { or } \\
\geq 0.3 \mathrm{mg} / \mathrm{dL} \text { within } 48 \mathrm{~h}\end{array}$ & \\
\hline Stage 2 & increase $\geq 100 \%$ & \\
\hline Stage 3 & increase $\geq 200 \%$ & \\
\hline KDIGO AKI criteria & serum creatinine & \\
\hline Stage 1 & $\begin{array}{c}\text { increase } \geq 50 \% \text { or } \\
\geq 0.3 \mathrm{mg} / \mathrm{dL} \text { within } 7 \text { days }\end{array}$ & \\
\hline Stage 2 & increase $\geq 100 \%$ & \\
\hline Stage 3 & $\begin{array}{c}\text { increase } \geq 200 \% \\
\text { or } \geq 35 \mathrm{~mL} / \mathrm{min} / 1.73 \mathrm{~m}^{2}\end{array}$ & \\
\hline
\end{tabular}


of pediatric HSCT and current diagnostic tools for its assessment. We also aimed to discuss new markers that may potentially be useful in the early diagnosis of HSCT-related AKI, although they are not yet used in clinical practice.

\section{Reasons for acute kidney injury following hematopoietic stem cell transplantation}

The first determinant of post-HSCT AKI is the type of HSCT itself. Myeloablative allogeneic HSCT is characterized by the highest incidence of AKI (50-91\%), whereas nonmyeloablative allogeneic (29-54\%) and autologous HSCT (12-52\%) are less frequently involved in AKI. ${ }^{13}$

The pathogenesis of AKI following HSCT is complex and covers pre-renal, renal and post-renal mechanisms. ${ }^{14}$ Among them, renal causes are the most common, with ischemia and acute tubular necrosis due to drug nephrotoxicity playing the main role. The causes of renal impairment after HSCT also strongly depend on the amount of time since the transplantation, and can be divided into immediate (tumor lysis syndrome, marrow infusion toxicity), early (hypovolemia due to diarrhea, vomiting and sepsis, drug nephrotoxicity, acute tubular necrosis, interstitial nephritis, reactivation of viral infections resulting in hemorrhagic cystitis, graft vs host disease (GvHD), and hepatic sinusoidal obstruction syndrome (HSOS)) and late (thrombotic microangiopathy, chronic GvHD and radiation). ${ }^{13}$

Describing the whole spectrum of HSCT-related factors predisposing to AKI is beyond the scope of this review; we are therefore going to focus only on selected ones. Among them, GvHD seems to be the best example of the multifactorial nature of HSCT-related AKI. ${ }^{13}$

\section{Graft vs host disease}

In the course of GvHD, the kidneys become targets for T cells. During the first month after HSCT, the process of infiltration of the tubulointerstitial area occurs. Immunocompetent cell migration and cytokine-mediated inflammatory reaction in situ result in damage to the peritubular capillaries, tubules and glomeruli, and in thickening of the vascular intima. ${ }^{15}$ Paradoxically, the prophylactic regimen against GvHD, containing cyclosporine, tacrolimus or short-term methotrexate, may become another trigger factor for AKI. Moreover, the indirect propensity towards reactivation of viral infection that arises in the course of GvHD adds to the AKI-promoting background. ${ }^{15}$

\section{Marrow transfusion toxicity}

Cryopreservation of stem cells is a standard component of the clinical protocol in autologous HSCT, whereas it is rare in allogeneic HSCT. The collected cells are washed and resuspended in a solution supplemented with a cryoprotective agent, most typically dimethyl sulfoxide (DMSO). Dimethyl sulfoxide is considered the main causal agent of adverse reactions, such as lysis of erythrocytes, leading to precipitation of heme proteins in the proximal tubules and subsequent acute tubular necrosis. ${ }^{16}$

\section{Hepatic sinusoidal obstruction syndrome}

The pathophysiology of HSOS, present mainly in patients who have undergone myeloablative allogeneic HSCT, remains poorly defined. Total body irradiation and busulfan/cyclophosphamide preconditioning can cause damage to sinusoidal endothelial cells and hepatocytes. ${ }^{17}$ The subsequent subendothelial deposition of morphological blood compounds and fibrin results in hepatic venular narrowing and occlusion; portal hypertension then evolves. Consequently, the accumulation of vasodilators in the liver results in systemic hypotension. The compensative activation of the renin-angiotensin-aldosterone system (RAAS) triggers constriction of renal vessels and hypoperfusion. ${ }^{17}$

\section{Infections}

The use of immunosuppressive drugs may lead to reactivation of latent viral infections, affecting approx. $25 \%$ of patients who have undergone allogeneic HSCT. ${ }^{18}$ The majority of the population is seropositive to the BK virus - a member of the polyomavirus family with tropism to the urinary tract. The reactivation and massive replication of the BK virus lead to the formation of decoy cells (cells with large intranuclear inclusion bodies) programmed to die and release copied viral components, and are implicated in the pathogenesis of hemorrhagic cystitis and urethral stenosis. BK viruria may escalate to viremia and finally to the presence of the BK virus in the tubular epithelial cells of the kidney, and to kidney damage in the course of tubulointerstitial nephritis.

Another potent infectious condition in the course of HSCT is sepsis, triggering both pre-renal (hypovolemia due to inflammatory vasodilation) and renal (direct tubular damage due to cytokine release) circumstances facilitating the development of AKI. 


\section{Transplant-associated thrombotic microangiopathy}

Transplant-associated thrombotic microangiopathy (TA-TMA) is in the spectrum of disorders characterized by vascular endothelial injury followed by arteriolar thrombi, intimal swelling and fibrinoid necrosis. Clinically, it is manifested by thrombocytopenia, hemolytic anemia and tissue hypoxia. Although TA-TMA may occur in any organ, the kidneys are affected in $40 \%$ of cases, and $20 \%$ of them undergo a severe course. The risk factors for TA-TMA include the use of pharmacotherapy with calcineurin inhibitors, acute GvHD, total body irradiation, patient-donor gender mismatch, and infections. ${ }^{19}$

\section{Nephrotoxicity of chemotherapy}

Potentially nephrotoxic drugs are used both during conditioning therapy (busulfan, treosulfan, fludarabine, thiotepa, melphalan, cyclophosphamide) and as GvHD prophylaxis (cyclosporin A, tacrolimus, methotrexate). ${ }^{20}$ The pathomechanisms of nephrotoxicity for calcineurin inhibitors are not fully defined. Cyclosporin A and tacrolimus activate the RAAS, leading to vasoconstriction of the renal afferent arterioles and subsequent AKI. They also increase oxidative stress, acting directly on renal endothelial cells. Finally, through increased production of vascular endothelial growth factor (VEGF), they trigger peritubular fibrosis, which is responsible for chronic drug-induced nephropathy. Methotrexate (MTX), especially in high doses, precipitates in acidic $\mathrm{pH}$ in the lumen of the renal tubules, leading to tubular toxicity. ${ }^{20}$ Male sex, low serum albumin levels, previous kidney pathologies, and interactions with other drugs (including furosemide) may intensify the nephrotoxicity of MTX.

The mechanism of the toxicity of cisplatin is attributed to its accumulation in the kidney, mainly in the epithelial cells of the proximal tubules, leading to their dysfunction. ${ }^{20}$ The epithelium of the distal tubules and glomeruli are subsequently affected. Cyclophosphamide has a high affinity to urothelium, so hemorrhagic cystitis is a major clinical manifestation of its usage. However, it also acts detrimentally on the renal tubules by inducing oxidative stress. Isophosphamide is characterized by tropism to the epithelium of the proximal tubules, causing AKI.

Drugs used in the prophylaxis or treatment of infections, such as aminoglycosides, amphotericin B or acyclovir, are well known for their nephrotoxic mechanisms. Amphotericin B acts through renal vasoconstriction and hypoxia of the proximal tubule cells; aminoglycosides accumulate directly in the proximal tubules, changing their permeability; whereas acyclovir precipitates in the tubules, causing their obstruction. ${ }^{20}$

\section{Markers of acute kidney injury}

The high incidence of AKI in the course of HSCT has engendered a search for sensitive and specific markers of kidney damage. Although serum creatinine-based equations remain the major tool for assessing kidney function by eGFR, there is need for more adequate parameters that would allow early diagnosis of AKI, preferably at the stage when it is still reversible. Since the proximal tubule is the first target cell in AKI-triggered renal damage, the research should focus mainly on markers that directly characterize its function.

\section{Parameters of tubular damage}

\section{Cystatin C}

Cystatin C (CysC) is a low molecular weight $(13 \mathrm{kDa})$ non-glycosylated protein generated by all nucleated cells, present in all tissues and physiological fluids. CysC can be assessed by ELISA in both serum and urine. Serum CysC outperforms serum creatinine in assessing eGFR in children. ${ }^{21}$ Increases in serum CysC have been shown to correlate with the development of AKI in children from intensive care units and following cardiac surgery, as well as in adults following HSCT. ${ }^{22}$ However, it has also been helpful in analyzing glomerular damage due to chemotherapy in children. ${ }^{23}$ Moreover, increased serum CysC concentrations have been observed in patients with leukemia, melanoma, as well as colorectal or hepatocellular cancer, but without renal injury. Therefore, high serum CysC is not specific for AKI, because it may well be a marker of malignancy. ${ }^{24}$

On the other hand, CysC is filtered by glomeruli, then reabsorbed and degraded in the proximal tubules. It is not secreted by the tubules, so its urinary concentration is minimal in subjects with normal kidney function, but increases significantly in the course of kidney injury. ${ }^{25}$ Therefore, urinary CysC may be a potential early marker of proximal tubule damage in the course of post-HSCT AKI, but no data are available.

\section{Neutrophil gelatinase-associated lipocalin}

Neutrophil gelatinase-associated lipocalin (NGAL) is another low-molecular-weight protein $(25 \mathrm{kDa})$, originally identified in secondary granules of human neutrophils and as such, is released into circulation from organs like the liver, lungs, trachea, colon, uterus, or prostate. It is filtered by the glomeruli and reabsorbed by the proximal tubules. ${ }^{26}$

Serum NGAL acts in a bacteriostatic manner by binding siderophores - bacterial products that scavenge iron. Kidney-derived NGAL accompanies the differentiation and development of the tubular epithelium and is one of the earliest markers of distal nephron damage. ${ }^{26}$ 
Both serum and urine NGAL are useful predictors of AKI, although its urinary concentration is more sensitive for kidney injury. ${ }^{27}$ NGAL is widely used for early diagnosis of AKI, its monitoring and prognosis. It has also become useful in pediatric AKI, mainly in its early diagnosis after cardiac surgery and as a predictor of mortality.

However, NGAL is also overexpressed in other conditions, such as tumors or inflammatory bowel disease. ${ }^{28}$ It is used to monitor the nephrotoxicity of drugs like iphosphamide, cisplatin and cyclosporine. ${ }^{29}$ Increased plasma NGAL reflects inflammation, including sepsis, whereas urinary NGAL points rather to urinary tract infection. ${ }^{30}$ NGAL may also suppress metastatic processes. ${ }^{28}$

The role of urinary NGAL in predicting AKI has been demonstrated in adults who have undergone HSCT for oncological reasons: as markers of AKI, elevated urinary NGAL preceded increased serum creatinine by 2 days. ${ }^{31}$ No such investigation has been performed in children who have undergone HSCT.

\section{Kidney injury molecule 1}

Kidney injury molecule 1 (KIM-1) is a glycoprotein, the localization of which is limited to the apical membranes of the proximal tubules. Physiological urinary KIM-1 concentration is negligible, whereas it increases after ischemic or nephrotoxic AKI. Renal biopsies performed after acute tubular necrosis revealed increased expression of KIM-1 in proximal tubule cells. ${ }^{32}$ This molecule is also a scavenger receptor of paramount importance for apoptosis and regeneration of damaged epithelial cells in the proximal tubules. Urinary KIM-1 has been shown to be useful in the detection of neoplasms originating in the proximal tubules, ovaries or uterus (clear cell cancer). ${ }^{28}$

Increases in urinary KIM-1 have been found to correlate with AKI due to acute tubular necrosis. ${ }^{33}$ In children, KIM-1 has been shown to be a useful marker for AKI following shock-related hypovolemia - more sensitive and specific than serum creatinine, NGAL or interleukin-18 (IL-18). However, the correlation with the severity of AKI in children from an intensive care unit was quite weak, although KIM-1 could strongly predict the need for renal replacement therapy. ${ }^{34}$ Kidney injury molecule 1 is also a useful marker of kidney function in children undergoing chemotherapy with MTX or cisplatin derivatives. ${ }^{35}$ Although the value of KIM-1 as a marker for AKI has been demonstrated in a recent meta-analysis, ${ }^{36}$ none of the data concerned the detection of AKI following HSCT.

\section{$\mathrm{N}$-acetyl-beta-D-glucosaminidase}

$\mathrm{N}$-acetyl-beta-D-glucosaminidase (NAG) is a lysosomal enzyme of high molecular weight, found originally in proximal tubule cells, released after their damage and therefore considered a marker of their dysfunction. The urinary concentration of NAG increases in AKI, as has been shown in adult patients that have undergone cardiac surgery. ${ }^{37}$ Like KIM-1, NAG is a strong predictor of a poor prognosis in adults with AKI. ${ }^{38}$

Preliminary reports from animal studies suggest that NAG may be useful in assessing AKI in the course of acute GvHD following allogeneic HSCT. ${ }^{39}$ Urinary NAG could detect tubular damage at the subclinical level and hepatic veno-occlusive disease, although it did not correlate with the level of azotemia in patients who had undergone allogeneic HSCT. ${ }^{40}$ Elevated urinary NAG has also been reported in subjects with acute tubular necrosis not related to HSCT. There are 2 recent studies on increased NAG concentration in the urine of adults and children who have undergone HSCT. ${ }^{41,42}$ These results are promising, because urinary NAG seems to be an ideal marker of early tubular damage, preceding the development of AKI.

\section{Inflammatory markers}

\section{Interleukin-18}

Interleukin 18 is a pro-inflammatory cytokine, released by monocytes/macrophages, activated by caspase- 1 and inducing interferon gamma. Widespread, IL-18 is also present in various kidney structures, like distal tubules, collecting ducts and the ascending limb of the loop of Henle. However, it is not found in proximal tubules or glomeruli. Increased serum IL-18 concentrations are typical of many inflammation-related diseases, including autoimmune disorders like lupus, arthritis, inflammatory bowel disease, psoriasis, hepatitis, diabetes, or sclerosis multiplex. ${ }^{43}$ This multiplicity suggests the weak specificity of this marker.

Animal studies have shown elevated urinary concentration of IL-18 in AKI following ureteral obstruction. ${ }^{44}$ Due to its pro-inflammatory activity, IL-18 seems to aggravate kidney damage in the course of ischemia/reperfusion injury; patients with acute tubular necrosis presented with higher urinary IL-18 than those with CKD, urinary tract infection, nephrotic syndrome, or pre-renal azotemia. ${ }^{45}$ Urinary IL-18 (like NGAL) has been found to be a strong predictor of AKI in children who had undergone cardiac surgery, since the diagnosis was made $12 \mathrm{~h}$ earlier than with serum creatinine. ${ }^{46}$ The prognostic value of IL-18 in diagnosing AKI, the need for renal replacement therapy and 90-day mortality was demonstrated in a large cohort of adults in an intensive care unit. ${ }^{47}$ However, there is still a need for thorough investigation of the applicability of IL-18 to detect AKI following HSCT in children.

\section{Monocyte chemoattractant protein 1}

Monocyte chemoattractant protein (MCP)- 1 is one of the key chemokines regulating the migration and infiltration of monocytes/macrophages to the sites of inflammation. 
It is also a pathogenic factor in many diseases, including AKI. Rice et al. noted an elevated urinary concentration of MCP-1 in mice at 24 and $72 \mathrm{~h}$ after renal ischemia. ${ }^{48}$ In another study, increased expression of MCP-1 in the renal cortex of mice with AKI correlated with the level of kidney damage better than NGAL. ${ }^{49}$ Urinary MCP-1 has been found to correlate strongly with the level of AKI in patients treated with cisplatin or tacrolimus, as well as with serum creatinine increase in adults 5 weeks after HSCT; it also predicted mortality risk after cardiac surgery. ${ }^{50}$ A significant elevation of MCP-1 concentration in children undergoing HSCT has been observed between the $2^{\text {nd }}$ and $4^{\text {th }}$ week after the procedure, but it was not correlated with AKI. ${ }^{51}$

\section{New acute kidney injury indexes}

\section{Liver-type fatty acid-binding protein}

Liver-type fatty acid-binding protein (L-FABP) is produced mainly in the liver, but its expression is also noticeable in other organs, including the kidneys. It takes part in the transport and metabolism of free fatty acids, acting as an anti-oxidant. In the proximal tubules, it allows intracellular metabolism of fatty acids. It is filtered by the glomeruli and reabsorbed by the proximal tubules. ${ }^{52}$ It is not present in the urine of healthy subjects, but has been found to be an early predictor of AKI due to sepsis or drug nephrotoxicity. ${ }^{53,54}$ Increased urinary concentrations of L-FABP have also been described in adults with acute decompensated heart failure or surgically treated aortic aneurysm. ${ }^{55,56}$ To date, a single promising report has been published on urinary L-FABP as a marker of AKI risk in adult patients following HSCT. ${ }^{57}$

\section{Insulin-like growth factor-binding protein 7}

Insulin-like growth factor-binding protein (IGFBP)-7 is another low-molecular-weight protein $(30 \mathrm{kDa})$ freely filtered by the glomeruli and reabsorbed by the proximal tubules; its increased urinary concentration is most probably the consequence of tubular damage. ${ }^{58}$ Indeed, increased urinary IGFBP-7 concentrations have been noticed in patients from intensive care units. They could also predict the renal outcome, distinguishing between patients with and without AKI, or between those with early recovery from AKI and late or non-recovery. ${ }^{59}$ Moreover, IGFBP-7 has been found to be a more useful tool than NGAL in the differential diagnosis and prognosis of AKI. Thus, IGFBP-7 seems another ideal candidate as a diagnostic and prognostic marker of AKI. However, no data is available on investigations of urinary IGFBP-7 concentrations in patients who have undergone HSCT, irrespective of their age.

\section{Netrin-1}

Netrin-1 is a $70 \mathrm{kDa}$ axon guidance molecule with an anti-inflammatory activity, expressed in many organs, including the kidneys. In vitro investigations have demonstrated its paramount role in ischemia/reperfusion kidney injury, during which the expression of netrin-1 increased in the apical membranes of proximal tubule cells, acting in a protective manner. Its anti-inflammatory and antiapoptotic activity has also been found to be highly efficient against cisplatin-induced kidney injury. ${ }^{60}$ Moreover, urinary netrin-1 has been reported to be an early predictor of AKI following cardiac surgery. ${ }^{61}$ However, no evidence for its role as a biomarker of HSCT-related AKI has been presented to date.

\section{Markers of acute kidney injury progression to chronic kidney disease}

Although potentially reversible, in some cases AKI progresses into CKD. Long-term unfavorable outcomes for children with AKI are defined in the pRIFLE criteria as loss of kidney function (L) and end-stage kidney disease (E). The major nephrological challenge is therefore to identify patients at risk of developing CKD after an episode of AKI. Candidate markers for assessing AKI-to-CKD progression include NGAL, KIM-1 and L-FABP, although convincing data on their utility in long-term observation of children who have undergone HSCT are lacking. ${ }^{62}$ Another promising biomarker is clusterin, identified in animal studies as a factor responsible for anti-inflammatory and anti-fibrotic activity after ischemia/reperfusion injury. ${ }^{63}$ However, tools for predicting AKI-to-CKD progression following HSCT are yet to be established.

\section{Targeted, pleiotropic or combined?}

Post-HSCT AKI has a multifactorial background, and as such should be assessed in a complex way. Many questions about the quantity and quality of markers used to evaluate $\mathrm{AKI}$ in these conditions remain unanswered. Can we rely on single markers specific for (e.g.) tubular damage, inflammation or drug side effects, or should we search for markers with pleiotropic functions, designating (e.g.) both tissue damage and nephrotoxicity? Is urine the only milieu that adequately shows AKI-related anomalies or should we implement serum markers as additional predictors? Is the early diagnosis of AKI our major goal or should we strive for both protectors of kidney function and predictors of renal outcomes? These questions also remain unanswered in the general field of AKI. However, 
some attempts have been made to establish new paradigms of AKI diagnosis, tailored to various pathologies responsible for kidney injury. Parikh and Mansour have suggested combinations of new urinary markers helpful in distinguishing between acute tubular necrosis and reversible prerenal azotemia due to hypoxia (IL-18, NGAL, KIM-1), or between the aforementioned conditions and hepatorenal syndrome (NGAL, L-FABP, IL-18). ${ }^{64}$ Another interesting option is the concomitant evaluation of markers in pairs, as in the case of association of [TIMP-2] $\times$ [IGFBP-7] in the urine of critically ill adults, where it has identified AKI patients at increased risk of mortality. ${ }^{65}$ These examples represent a promising direction, but more studies are needed to establish guidelines applicable in everyday practice, including guidelines for patients undergoing HSCT.

\section{Clinical perspective}

No guidelines for the early detection of AKI in children undergoing HSCT exist. Preliminary data suggest the usefulness of urinary NGAL and L-FABP in adults following HSCT, and urinary NAG has been positively tested in children following HSCT. However, the use of a marker in pediatric clinical practice demands easily available material and accessible age-related reference values. Currently, the data on the latter involve established markers of AKI, such as urinary IL-18, KIM-1, L-FABP, and NGAL. ${ }^{66}$ Fortunately, this combination additionally covers differential diagnostics, essential in cases of HSCT-related AKI, between pre-renal azotemia, acute tubular necrosis and liver-based causes of AKI. Moreover, KIM-1, L-FABP and NGAL may predict AKI-to-CKD progression. Therefore, this quartet appears to be the most promising and should be recommended first for clinical testing in children who have undergone HSCT.

\section{Conclusions}

Acute kidney injury is becoming a serious clinical challenge in the era of widespread HSCT availability. The panel of candidate AKI markers is wide, although the majority of them have not yet been tested in post-HSCT conditions. It is clear that the analysis of diagnostic and prognostic tools for AKI in children who have undergone HSCT should take into account the multifactorial nature of the process, including hypoxia, drug toxicity and immune-mediated reactions in the course of GvHD. Although none of the AKI biomarkers discussed in this review is used in everyday clinical practice, urinary IL-18, KIM-1, L-FABP, and NGAL seem like promising candidates to be tested in the pediatric population undergoing HSCT. From a clinical point of view, a reliable panel of AKI markers is an emerging necessity, if the therapy introduced today is to be effective tomorrow.

\section{References}

1. Kizilbash SJ, Kashtan CE, Chavers BM, Cao Q, Smith AR. Acute kidney injury and the risk of mortality in children undergoing hematopoietic stem cell transplantation. Biol Blood Marrow Transplant. 2016;22(7):1264-1270.

2. Ileri T, Ertem M, Ozcakar ZB, et al. Prospective evaluation of acute and chronic renal function in children following matched related donor hematopoietic stem cell transplantation. Pediatr Transplant. 2010;14(1):138-144.

3. Didsbury MS, Mackie FE, Kennedy SE. A systematic review of acute kidney injury in pediatric allogeneic hematopoietic stem cell recipients. Pediatr Transplant. 2015;19(5):460-470.

4. Raina R, Herrera N, Krishnappa V, et al. Hematopoietic stem cell transplantation and acute kidney injury in children: A comprehensive review. Pediatr Transplant. 2017;21(4):e12935.

5. Koh K-N, Sunkara A, Kang G, et al. Acute kidney injury in pediatric patients receiving allogeneic hematopoietic cell transplantation: Incidence, risk factors, and outcomes. Biol Blood Marrow Transplant. 2018;24(4):758-764.

6. Bellomo R, Ronco C, Kellum JA, Mehta RL, Palevsky P. Acute Dialysis Quality Initiative workgroup: Acute renal failure - definition, outcome measures, animal models, fluid therapy and information technology needs: The Second International Consensus Conference of the Acute Dialysis Quality Initiative (ADQI) Group. Crit Care. 2004; 8(4):R204-R212.

7. Mehta RL, Kellum JA, Shah SV, et al; Acute Kidney Injury Network. Acute Kidney Injury Network: Report of an initiative to improve outcomes in acute kidney injury. Crit Care. 2007;11(2):R31.

8. Kidney Disease: Improving Global Outcomes (KDIGO) Acute Kidney Injury Work Group. KDIGO Clinical Practice Guideline for Acute Kidney Injury. Kidney Int Suppl. 2012;2:1-138.

9. Sutherland SM, Byrnes JJ, Kothari M, et al. AKI in hospitalized children: Comparing the pRIFLE, AKIN, and KDIGO definitions. Clin J Am Soc Nephrol. 2015;10(4):554-561.

10. Holmes J, Roberts G, May K, et al. The incidence of pediatric acute kidney injury is increased when identified by a change in a creatinine-based electronic alert. Kidney Int. 2017;92(2):432-439.

11. Laskin BL, Nehus E, Goebel J, Furth S, Davies SM, Jodele S. Estimated versus measured glomerular filtration rate in children before hematopoietic cell transplantation. Biol Blood Marrow Transplant. 2014; 20(12):2056-2061.

12. Schwartz GJ, Munoz A, Schneider MF, et al. New equations to estimate GFR in children with CKD. J Am Soc Nephrol. 2009;20(3): 629-637.

13. Kemmner S, Verbeek M, Heemann U. Renal dysfunction following bone marrow transplantation. J Nephrol. 2017;30(2):201-209.

14. Lopes J, Jorge S, Neves M. Acute kidney injury in HCT: An update. Bone Marrow Transplant. 2016;51:755-762.

15. Motoyoshi Y, Endo A, Takagi M, et al. Graft versus host disease-dependent renal dysfunction after hematopoietic stem cell transplantation. CEN Case Rep. 2014;3(2):202-205.

16. Shu Z, Heimfeld S, Gao D. Hematopoietic SCT with cryopreserved grafts: Adverse reactions after transplantation and cryoprotectant removal before infusion. Bone Marrow Transplant. 2014;49:469-476.

17. Fan CQ, Crawford JM. Sinusoidal obstruction syndrome (hepatic veno-occlusive disease). J Clin Exp Hepatol. 2014;4(4):332-346.

18. Abudayyeh A, Hamdi A, Lin H, et al. Symptomatic BK virus infection is associated with kidney function decline and poor overall survival in allogeneic hematopoietic stem cell recipients HHS Public Access. Am J Transplant. 2016;16(5):1492-1502.

19. Rosenthal J. Hematopoietic cell transplantation-associated thrombotic microangiopathy: A review of pathophysiology, diagnosis, and treatment. J Blood Med. 2016;7:181-186.

20. Perazella MA. Onco-nephrology: Renal toxicities of chemotherapeutic agents. Clin J Am Soc Nephrol. 2012;7(10):1713-1721.

21. Nakhjavan-Shahraki B, Yousefifard M, Ataei N, et al. Accuracy of cystatin $C$ in prediction of acute kidney injury in children; serum or urine levels: Which one works better? A systematic review and meta-analysis. BMC Nephrol. 2017;18(1):120.

22. Volpon LC, Sugo EK, Carlotti APCP. Diagnostic and prognostic value of serum cystatin $C$ in critically ill children with acute kidney injury. Pediatr Crit Care Med. 2015;16(5):e125-131. 
23. Kos J, Lah TT. Cysteine proteinases and their endogenous inhibitors: Target proteins for prognosis, diagnosis and therapy in cancer (review). Oncol Rep. 1998;5(6):1349-1361.

24. Lah TT, Kos J. Cysteine proteinases in cancer progression and their clinical relevance for prognosis. Biol Chem. 1998;379:125-130.

25. Muto H, Ohashi K, Ando M, Akiyama H, Sakamaki H. Cystatin C level as a marker of renal function in allogeneic hematopoietic stem cell transplantation. Int J Hematol. 2010;91(3):471-477.

26. Schmidt-Ott KM, Mori K, Li JY, et al. Dual action of neutrophil gelatinase-associated lipocalin. J Am Soc Nephrol. 2007;18(2):407-413.

27. Abdulaziz Kari J, Ahmed Shalaby M, Sofyani K, et al. Urinary neutrophil gelatinase-associated lipocalin (NGAL) and serum cystatin $C$ measurements for early diagnosis of acute kidney injury in children admitted to PICU. World J Pediatr. 2018;14(2):134-142.

28. Marchewka Z, Tacik A, Piwowar A. KIM-1 and NGAL as potential biomarkers for the diagnosis and cancer progression. Postepy Hig Med Dosw. 2016;70:329-336.

29. Wasilewska A, Zoch-Zwierz W, Taranta-Janusz K, Michaluk-Skutnik J. Neutrophil gelatinase-associated lipocalin (NGAL): A new marker of cyclosporine nephrotoxicity? Pediatr Nephrol. 2010;25(5):889-897.

30. Soni SS, Cruz D, Bobek I, et al. NGAL: A biomarker of acute kidney injury and other systemic conditions. Int Urol Nephrol. 2010;42(1): 141-150.

31. Taghizadeh-Ghehi M, Sarayani A, Ashouri A, Ataei S, Moslehi A, Hadjibabaie M. Urine neutrophil gelatinase associated lipocalin as an early marker of acute kidney injury in hematopoietic stem cell transplantation patients. Ren Fail. 2015;37(6):994-998.

32. Han WK, Bailly V, Abichandani R, Thadhani R, Bonventre JV. Kidney Injury Molecule-1 (KIM-1): A novel biomarker for human renal proximal tubule injury. Kidney Int. 2002;62:237-244.

33. Huang Y, Don-Wauchope AC. The clinical utility of kidney injury molecule-1 in the prediction, diagnosis and prognosis of acute kidney injury: A systematic review. Inflamm Allergy Drug Targets. 2011;10(4): 260-271.

34. Westhoff JH, Seibert FS, Waldherr S, et al. Urinary calprotectin, kidney injury molecule-1, and neutrophil gelatinase-associated lipocalin for the prediction of adverse outcome in pediatric acute kidney injury. Eur J Pediatr. 2017;176(6):745-755.

35. Carvalho Pedrosa D, Macedo De Oliveira Neves F, Meneses GC, et al. Urinary $\mathrm{KIM}-1$ in children undergoing nephrotoxic antineoplastic treatment: A prospective cohort study. Pediatr Nephrol. 2015;30(12): 2207-2213.

36. Shao X, Tian L, Xu W, et al. Diagnostic value of urinary kidney injury molecule 1 for acute kidney injury: A meta-analysis. PLoS One. 2014;9(1):e84131.

37. Lannemyr L, Lundin E, Reinsfelt B, et al. Renal tubular injury during cardiopulmonary bypass as assessed by urinary release of $\mathrm{N}$-acetylß-D-glucosaminidase. Acta Anaesthesiol Scand. 2017;61(9):1075-1083.

38. Liangos $\mathrm{O}$, Perianayagam MC, Vaidya VS, et al. Urinary N-acetyl-beta(D)-glucosaminidase activity and kidney injury molecule-1 level are associated with adverse outcomes in acute renal failure. J Am Soc Nephrol. 2007;18(3):904-912.

39. Koyner JL, Shaw AD, Chawla LS, et al. Tissue inhibitor metalloproteinase-2 (TIMP-2) and IGF-binding protein-7 (IGFBP7) levels are associated with adverse long-term outcomes in patients with AKI. J Am Soc Nephrol. 2015;26(7):1747-1754

40. Fink J, Cooper M, Burkhart K, McDonald G, Zagerr Fink RJ, McDonald GB, Zager RA. Marked enzymuria after bone marrow transplantation: A correlate of veno-occiusive disease-induced hepatorenal syndrome. J Am Soc Nephrol. 1995;6(6):1655-1656.

41. Volkan Hazar MD, Ozgul Gungor MD, Ayfer Gur Guven MD, et al. Renal function after hematopoietic stem cell transplantation in children. Pediatr Blood Cancer.2009;53(2):97-202.

42. Morito T, Ando M, Tsuchiya K, Nitta K. Early identification of acute kidney injury after hematopoietic stem cell transplantation by the measurement of urinary biomarkers [in Japanese]. Nihon Jinzo Gakkai Shi. 2011;53(8):1150-1158.

43. Urbschat A, Obermüller N, Haferkamp A. Biomarkers of kidney injury. Biomarkers. 2011;16(Suppl 1):22-30.

44. Franke El, Vanderbrink BA, Hile KL, et al. Renal IL-18 production is macrophage independent during obstructive injury. PLoS One. 2012;7(10): e47417.
45. Wu H, Craft ML, Wang P, et al. IL-18 contributes to renal damage after ischemia-reperfusion. J Am Soc Nephrol. 2008;19(12):2331-2341.

46. Parikh CR, Mishra J, Thiessen-Philbrook H, et al. Urinary IL-18 is an early predictive biomarker of acute kidney injury after cardiac surgery. Kidney Int. 2006;70(1):199-203.

47. Nisula S, Yang R, Poukkanen M, et al. Predictive value of urine interleukin-18 in the evolution and outcome of acute kidney injury in critically ill adult patients. Br J Anaesth. 2015;114(3):460-468.

48. Rice JC, Spence JS, Yetman DL, Safirstein RL. Monocyte chemoattractant protein-1 expression correlates with monocyte infiltration in the post-ischemic kidney. Ren Fail. 2002;24(6):703-723.

49. Munshi R, Johnson $A$, Siew ED, et al. MCP-1 gene activation marks acute kidney injury. J Am Soc Nephrol. 2011;22(1):165-175.

50. Moledina DG, Isguven S, McArthur E, et al. Plasma monocyte chemotactic protein-1 is associated with acute kidney injury and death after cardiac operations. Ann Thorac Surg. 2017;104(2):613-620.

51. Dicarlo J, Agarwal-Hashmi R, Shah A, et al. Cytokine and chemokine patterns across 100 days after hematopoietic stem cell transplantation in children. Biol Blood Marrow Transpl. 2014;20(3):361-369.

52. Chmurzyńska $A$. The multigene family of fatty acid-binding proteins (FABPs): Function, structure and polymorphism. J App/ Genet. 2006; 47(1):39-48.

53. Doi K, Noiri E, Maeda-Mamiya R, et al. Urinary L-type fatty acid-binding protein as a new biomarker of sepsis complicated with acute kidney injury. Crit Care Med. 2010;38(10):2037-2042.

54. Negishi K, Noiri E, Sugaya T, et al. A role of liver fatty acid-binding protein in cisplatin-induced acute renal failure. Kidney Int. 2007;72(3): 348-358.

55. Hishikari K, Hikita H, Nakamura S, et al. Urinary liver-type fatty acidbinding protein level as a predictive biomarker of acute kidney injury in patients with acute decompensated heart failure. Cardiorenal Med. 2017;7(4):267-275.

56. Obata Y, Kamijo-Ikemori A, Ichikawa D, et al. Clinical usefulness of urinary liver-type fatty-acid-binding protein as a perioperative marker of acute kidney injury in patients undergoing endovascular or openabdominal aortic aneurysm repair. J Anesth. 2016;30(1):89-99.

57. Shingai N, Morito T, Najima Y, et al. Urinary liver-type fatty acid-binding protein linked with increased risk of acute kidney injury after allogeneic stem cell transplantation. Biol Blood Marrow Transplant. 2014;20(12):2010-2014.

58. Emlet DR, Pastor-Soler N, Marciszyn A, et al. Insulin-like growth factor binding protein 7 and tissue inhibitor of metalloproteinases-2: Differential expression and secretion in human kidney tubule cells. Am J Physiol Renal Physiol. 2017;312(2):F284-F296.

59. Aregger F, Uehlinger DE, Witowski J, et al. Identification of IGFBP-7 by urinary proteomics as a novel prognostic marker in early acute kidney injury. Kidney Int. 2014;85(4):909-919.

60. Rajasundari A, Pays L, Mehlen P, Ramesh G. Netrin-1 overexpression in kidney proximal tubular epithelium ameliorates cisplatin nephrotoxicity. Lab Investig. 2011;91(12):1717-1726.

61. Ramesh G, Krawczeski CD, Woo JG, Wang Y, Devarajan P. Urinary netrin-1 is an early predictive biomarker of acute kidney injury after cardiac surgery. Clin J Am Soc Nephrol. 2010;5(3):395-401.

62. Goldstein SL, Devarajan P. Acute kidney injury in childhood: Should we be worried about progression to CKD? Pediatr Nephrol. 2011;26(4): 509-522.

63. Guo J, Guan Q, Liu X, et al. Relationship of clusterin with renal inflammation and fibrosis after the recovery phase of ischemia-reperfusion injury. BMC Nephrol. 2016;17(1):133.

64. Parikh CR, Mansour SG. Perspective on clinical application of biomarkers in AKI. J Am Soc Nephrol. 2017;28(6):1677-1685

65. Koyner JL, Coca SG, Thiessen-Philbrook H, et al. Urine biomarkers and perioperative acute kidney injury: The impact of preoperative estimated GFR. Am J Kidney Dis. 2015;66(6):1006-1014.

66. Bennett MR, Nehus E, Haffner Ch, Ma Q, Devarajan P. Pediatric reference ranges for acute kidney injury biomarkers. Pediatr Nephrol. 2015;30(4):677-685. 